\title{
The effect of an encapsulated fatty acid mixture on the fatty acid composition of mares' milk
} M D Scott ${ }^{1}$, V E Beattie ${ }^{1}$, L M Katz ${ }^{2}$

${ }^{1}$ Devenish Nutrition Ltd, Belfast, United Kingdom, ${ }^{2}$ University College Dublin, Dublin, Ireland

Email: marian.scott@devenishnutrition.com

Introduction Milk production and composition, including fatty acid composition, may change in response to the effects of lactation stage, age, parity and nutrition (Doreau et al. 1992; Csapó et al. 1995). Fatty acid content, chain length and saturation in mares' milk is thought to be influenced directly by dietary content as little or no hydrogenation of unsaturated fatty acids is believed to occur prior to absorption in hind gut fermenting animals (Hoffman et al., 1998). The aim of the study was to compare the different fatty acid concentrations in mares' milk prior to and post inclusion of a fatty acid mixture and to evaluate how long it takes for the fatty acid profile of the milk to change.

Materials and methods 16 adult lactating thoroughbred mares maintained at pasture and at least 4 weeks post-foaling were included in the study. Prior to entering the study, all mares underwent a complete physical, haematological and biochemical evaluation as well as faecal analysis for parasites to assess their general health, with any animal showing physical and laboratory evidence of disease excluded from the study. All mares were then weighed and treated with the encapsulated fatty acid mixture (40g/day orally) for a total of 2 weeks. Approximately 30 mls of milk was collected from each mare prior to treatment and then after 1 and 2 weeks on treatment. Milk samples were stored at $-20^{\circ} \mathrm{C}$ until transported to the laboratory to be analysed. Milk fatty acids were prepared using methyl ester according to the method outlined in Amer et al. (1995) and the results were expressed as grams lipid/100 ml milk and \% of total fatty acids. Data was analysed using a one-way repeated measures ANOVA. Milk data was analysed taking into account the stage of lactation and the period at pasture for each individual mare as these factors can influence the fatty acid content of the milk.

Results 16 mares were included in the study with the average post-foaling days for the mares prior to entering the study $50.6 \pm 6.9$ SEM (range 16-114). The level of fatty acids present in the milk varied widely, with higher levels of long chain fatty acids being observed in comparison to short/medium chain fatty acids in all milk samples analysed. The encapsulated fatty acid mixture treatment resulted in increased levels of saturation, especially within the medium chain fatty acids (Table 1). The levels of unsaturated fatty acids observed remained consistent or decreased during the treatment period (Table 2). The observed increases in the medium chain saturated fatty acid content resulted in a subsequent decrease in long chain unsaturated content expressed as \% of total fatty acids with the exception of C18:3. The numerical increase in C18:3 may be a result of the mares being at pasture resulting in an increased source of unsaturated long chain fatty acids.

Table 1 Effect of treatment and time on saturated fatty acid content of mares milk (\% of total fatty acids)

\begin{tabular}{llcccccc}
\hline \hline & Total Fat $(\mathrm{g} / 100 \mathrm{ml}$ milk $)$ & $\mathrm{C} 4$ & $\mathrm{C} 8$ & $\mathrm{C} 10$ & $\mathrm{C} 12$ & $\mathrm{C} 14$ & $\mathrm{C} 16$ \\
\hline Prior & 2.5 & 0.05 & 2.6 & 5.7 & 6.1 & 5.9 & 19.6 \\
Week 1 & 2.1 & 0.08 & 2.9 & 6.6 & 7.7 & 7.3 & 20.1 \\
Week 2 & 1.9 & 0.06 & 2.7 & 5.7 & 6.4 & 6.3 & 19.7 \\
\hline P & NS & NS & 0.08 & $<0.01$ & 0.02 & NS \\
\hline \hline
\end{tabular}

Table 2 Effect of treatment and time on unsaturated fatty acid content of mares milk (\% of total fatty acids)

\begin{tabular}{lllllllll}
\hline \hline & Total Fat (g/100ml milk) & C15:1 & C16:1 & C17:1 & C18:1 & C18:3 & C20:1 & C20:2 \\
\hline Prior & 2.5 & 0.15 & 0.40 & 0.55 & 19.7 & 18.2 & 0.37 & 0.30 \\
Week 1 & 2.1 & 0.14 & 0.27 & 0.56 & 16.9 & 18.6 & 0.36 & 0.22 \\
Week 2 & 1.9 & 0.17 & 0.37 & 0.55 & 18.5 & 20.8 & 0.35 & 0.25 \\
\hline P & & 0.05 & $<0.05$ & NS & $<0.05$ & NS & NS & 0.06 \\
\hline \hline
\end{tabular}

Conclusions From the results obtained in this study it could be concluded that encapsulated fatty acids within the diet have the potential to enrich the mares' milk, even after only 1 week of supplementation. Reductions were observed in some of the fatty acids due to the modifications achieved. Fatty acids, especially short chain fatty acids, have been shown to be the preferred energy substrate for microbiota and may have a role in preventing certain types of colitis (Scheppach, 1994). Since intestinal microbiota are essential for the development of a healthy and stable intestinal tract/immune system in immature animals, modification of the fatty acid components of the mares milk may have the potential to impact foal health.

\section{References}

Amer, M. A., D. B. Kupranycez, and B. E. Baker. 1985. Journal of the American Oil Chemists' Society 62, $1551-1557$

Csapó J., Stefler J., Martin T. G., Makray S., Csapó-Kiss Z.1995. International Dairy Journal 5, 393-402

Doreau M., Boulot S., Bauchart D., Barlet J.-P., Martin-Rosset W. 1992. Journal of Nutrition 122, 992-999

Hoffman, R.H., Kronfeld, D.S., Herbein J.H., Swecker W.S., Cooper, W.L. and Harris, P.A. 1998. Journal of Nutrition 128, 2708S-2711S.

Scheppach, W. 1994. Gut 35, S35-S38. 